\title{
Do You Feel My Pain? Racial Group Membership Modulates Empathic Neural Responses
}

\author{
Xiaojing Xu, ${ }^{1}$ Xiangyu Zuo, ${ }^{1}$ Xiaoying Wang, ${ }^{2}$ and Shihui Han ${ }^{1}$ \\ 'Department of Psychology, Peking University, Beijing 100871, and 2Department of Radiology, Peking University First Hospital, Beijing 100034, People's \\ Republic of China
}

The pain matrix including the anterior cingulate cortex (ACC) mediates not only first person pain experience but also empathy for others' pain. It remains unknown, however, whether empathic neural responses of the pain matrix are modulated by racial in-group/out-group relationship. Using functional magnetic resonance imaging we demonstrate that, whereas painful stimulations applied to racial in-group faces induced increased activations in the ACC and inferior frontal/insula cortex in both Caucasians and Chinese, the empathic neural response in the ACC decreased significantly when participants viewed faces of other races. Our findings uncover neural mechanisms of an empathic bias toward racial in-group members.

\section{Introduction}

Empathy refers to the ability to understand and share others' emotion and plays a key role in social behaviors. Perception of others in pain or distress generates empathic concerns that provide a proximate mechanism selected by evolution that motivates altruistic behaviors (Batson, 1991; de Waal, 2008). Empathy may influence social behaviors by changing people's attitudes toward a target (Batson et al., 1997a), which sometimes produces serious consequences such as when making judicial decisions on a defendant (Johnson et al., 2002).

The perception-action model of empathy proposes that empathic responses do not require conscious and effortful processing and often occur automatically (Preston and de Waal, 2002). Consistent with this, neuroimaging studies have shown that perception of others in pain activates the neural circuit consisting of the anterior cingulate cortex (ACC) and insula that mediate firstperson pain experience (Singer et al., 2004; Botvinick et al., 2005; Jackson et al., 2005; Saarela et al., 2007). However, the empathic neural responses are modulated by affective link between individuals (Singer et al., 2006) and top-down attention to painful cues in stimuli (Gu and Han, 2007; Fan and Han, 2008). In addition, empathy may be influenced by social relationship between individuals such that empathic concerns increase if a perceiver and a target share common membership in a social category (Hornstein, 1978). The evidence supporting this hypothesis comes from research that measured subjective reports of empathic concern. Johnson et al. (2002) asked White university students to read a passage involving a Black or a White man who was charged with a criminal act. Participants were induced to feel no empathy, low empathy, or high empathy for the defendant and

Received May 23, 2009; accepted May 30, 2009.

This work was supported by National Natural Science Foundation of China (Project 30630025, 30828012).

Correspondence should be addressed to Dr. Shihui Han, Department of Psychology, Peking University, 5 Yiheyuan Road, Beijing 100871, People's Republic of China. E-mail: shan@pku.edu.cn.

DOI:10.1523/JNEUROSCI.2418-09.2009

Copyright @ 2009 Society for Neuroscience $\quad$ 0270-6474/09/298525-05\$15.00/0 then evaluate punishments applied to the defendant. Johnson et al. (2002) found that White participants reported greater feelings of empathy for and assigned more lenient punishments to the White than the Black defendant, suggesting an empathic bias toward racial in-group members.

The current work investigated the neural mechanism underlying modulations of empathic neural responses by racial group membership between individuals. We scanned Caucasian and Chinese participants using functional magnetic resonance imaging (fMRI) while they watched video clips of Caucasian or Chinese faces receiving painful stimulation (needle penetration) or non-painful stimulation (cotton Q-tip touch). Automatic categorization of others by race defines the intragroup or intergroup relations between a perceiver and the target. Our recent research (Han et al., 2009) found that, relative to Q-tip touch, needle penetration applied to faces of Chinese models with neutral expressions induced increased activations in the ACC and bilateral frontal cortices of Chinese participants. The present study further tested the hypothesis that the empathic neural responses are weakened by race-defined intergroup relationship and such effect is independent of perceivers' own race.

\section{Materials and Methods}

Subjects. Seventeen Chinese ( 8 males, mean $=23$ years, $\mathrm{SD}=2.0$ years, all right handed) and 16 Caucasian healthy college students $(8$ males, mean $=23$ years, $\mathrm{SD}=3.7$ years, 10 Americans, 2 Dutch, 1 Italian, 1 German, 1 Russian, 1 Israeli, 12 right handed, 4 left handed) were paid for participation. All had normal or corrected-to-normal vision and reported no abnormal neurological history. Informed consent was obtained from all participants before scanning. This study was approved by a local ethics committee.

Stimuli and procedure. The stimuli consisted of 48 video clips showing faces of six Chinese ( 3 males) and six Caucasian models ( 3 males). Each clip, subtending a visual angle of $21^{\circ} \times 17^{\circ}$ (width $\times$ height) at a viewing distance of $80 \mathrm{~cm}$, lasted $3 \mathrm{~s}$ and depicted a face with neutral expressions receiving painful (needle penetration) or non-painful (Q-tip touch) stimulation (Fig. 1a,b) applied to the left or right cheeks. After each video clip, participants were instructed to judge whether or not the model was 


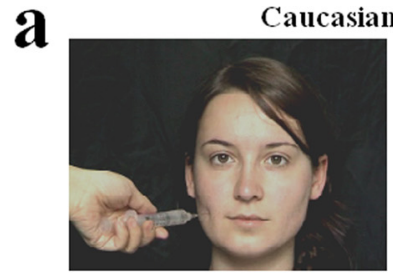

painful

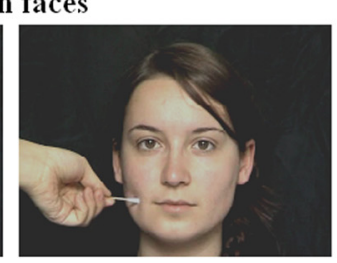

non-painful

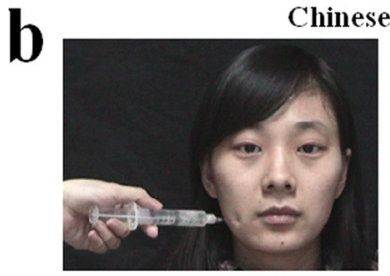

painful

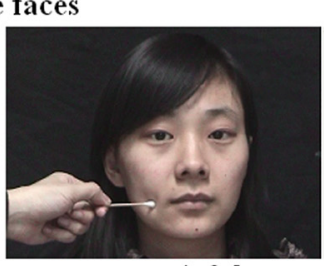

non-painful

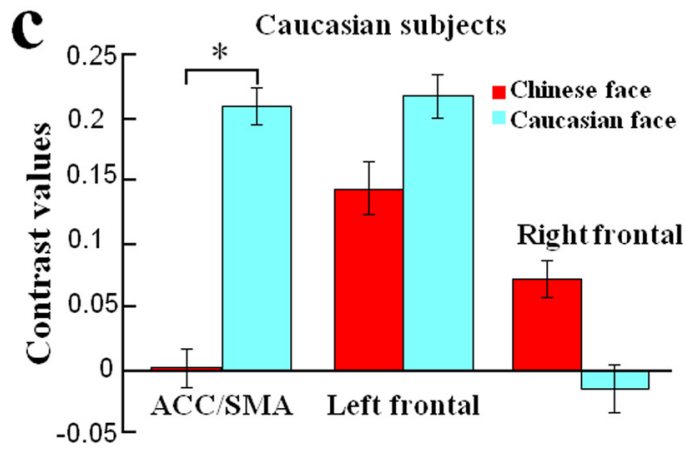

d
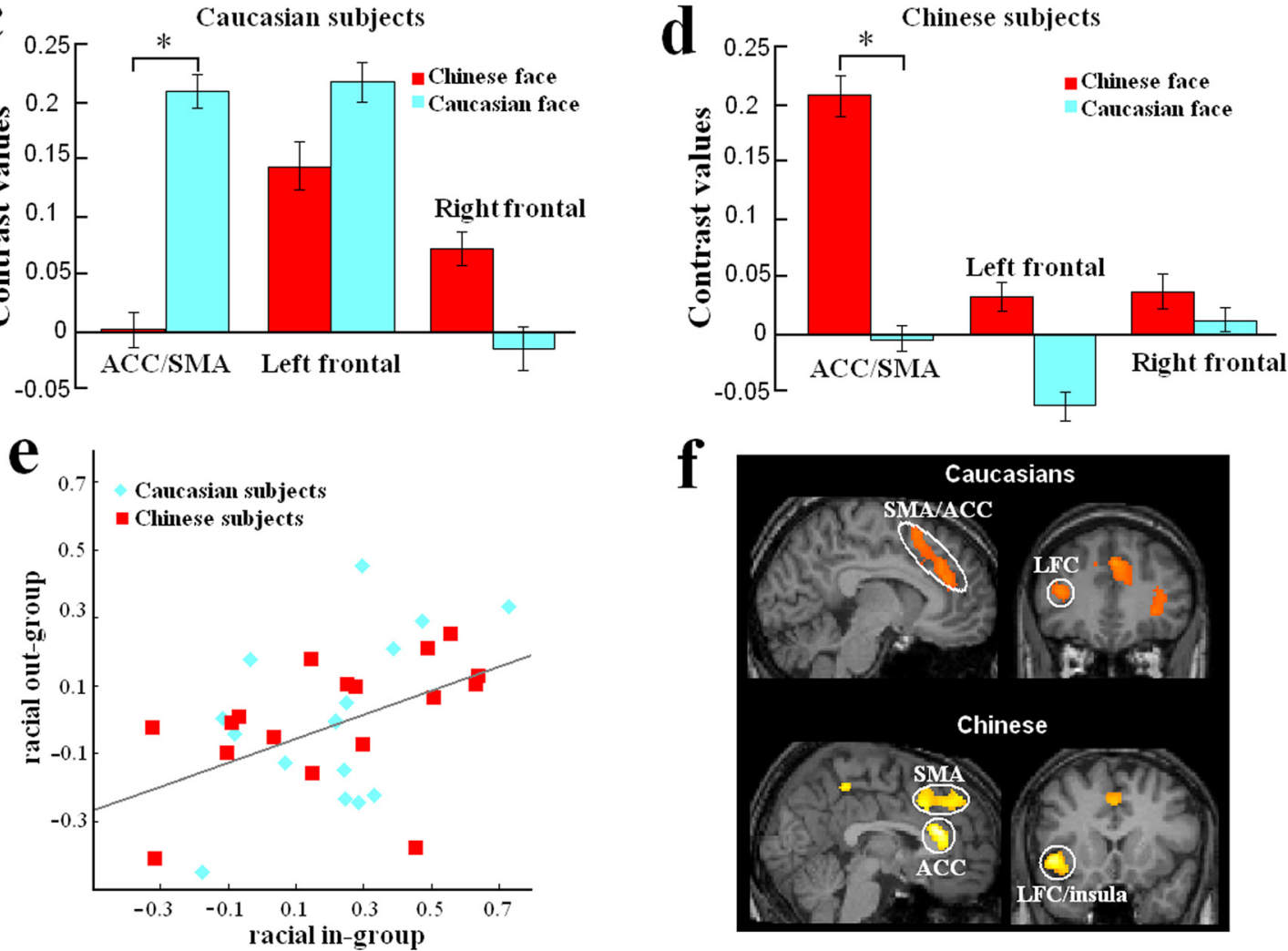

Figure 1. $\quad \boldsymbol{a}$, Illustration of Caucasian faces receiving painful and non-painful stimuli. $\boldsymbol{b}$, Illustration of Chinese faces receiving painful and non-painful stimuli. $\boldsymbol{c}$, Contrast values of the parameter estimates of signal intensity in the ACC and the frontal cortex that differentiated painful and non-painful stimuli in Caucasians. $\boldsymbol{d}$, Contrast values of the parameter estimates of signal intensity in the ACC and the frontal cortex that differentiated painful and non-painful stimuli in Chinese. $\boldsymbol{e}$, Correlation between ACC empathic neural responses to racial in-group and out-group members. $X$ and $Y$ axes respectively indicate $A C C$ empathic responses to racial in-group and racial out-group members indexed in contrast values of painful versus non-painful stimulation. $f$, Increased activations in the ACC and the frontal/insula cortex shown in whole-brain statistical parametric mapping analyses when participants perceived racial in-group faces. The upper figures show the results from Caucasian subjects and the lower figures show the results from Chinese subjects.

feeling pain by pressing a button using the right index or middle finger. Six functional scans of 204 s were obtained from each subject. Each scan consisted of 16 video clips (8 Chinese and 8 Caucasian faces, half with painful and half with non-painful stimulations in a random order). The interstimulus interval between two successive clips lasted $9 \mathrm{~s}$ during which participants fixated at a central cross. The last clip in each scan was followed by a fixation of $12 \mathrm{~s}$.

After the scanning procedure, participants were shown half of the video clips again and had to rate the pain intensity felt by the model ("How painful do you think the model feels?") and the unpleasantness felt by the onlooker ("How unpleasant do you feel when observing the video clip?") using a Likert-type scale where 0 indicated no effect and 10 indicated maximal effect (e.g., extremely painful, extremely unpleasant). Individuals' attitudes of ethnic identity were assessed using the Multigroup Ethnic Identity Measure (Phinney, 1992) (1 = strongly disagree; $4=$ strongly agree). The degree of endorsement of individualistic and collectivistic values was estimated using a 7-point Likert-type scale (Triandis and Gelfand, 1998) ( 1 = strongly disagree; $7=$ strongly agree). Individual differences in empathy ability were measured using the Empathic Concern Scale (Davis, 1996).

fMRI image acquisition and analysis. Scanning was performed at Pe- king University First Hospital, using a GE 3-T scanner with a standard head coil. Thirty-two transverse slices of functional images covering the whole brain were acquired using a gradient-echo echo-planar pulse sequence [ $64 \times 64 \times 32$ matrix with a spatial resolution of $3.75 \times 3.75 \times$ $4 \mathrm{~mm}$, repetition time $(\mathrm{TR})=3000 \mathrm{~ms}$, echo time $(\mathrm{TE})=30 \mathrm{~ms}$, field of view $(\mathrm{FOV})=24 \times 24 \mathrm{~cm}$, flip angle $\left.=90^{\circ}\right]$. Anatomical images were obtained using a 3 D FSPGR T1 sequence $(256 \times 256 \times 128$ matrix with a spatial resolution of $0.938 \times 0.938 \times 1.4 \mathrm{~mm}, \mathrm{TR}=7.4 \mathrm{~ms}$, inversion time $(\mathrm{TI})=450 \mathrm{~ms}, \mathrm{TE}=3.0 \mathrm{~ms}$, flip angle $=20^{\circ}$ ).

SPM2 (the Wellcome Trust Centre for Neuroimaging, London, UK) was used for fMRI data analysis. The functional data were first timecorrected to compensate for delays associated with acquisition time differences between slices during the sequential imaging. The functional images were then realigned to the first scan to correct for head motion between scans. The anatomical image was coregistered with the mean functional image produced during the process of realignment. All images were normalized to a $2 \times 2 \times 2 \mathrm{~mm}^{3}$ Montreal Neurological Institute (MNI) template. Functional images were spatially smoothed using a Gaussian filter with the full-width/half-maximum parameter (FWHM) set to $8 \mathrm{~mm}$ and temporally filtered using a cutoff of $128 \mathrm{~s}$. The event- 
Table 1. Mean rating scores (SD) of pain intensity and self-unpleasantness

\begin{tabular}{|c|c|c|c|c|}
\hline \multirow[b]{2}{*}{ Video types } & \multicolumn{2}{|l|}{ Chinese face } & \multicolumn{2}{|c|}{ Caucasian face } \\
\hline & Needle & Q-tip & Needle & Q-tip \\
\hline \multicolumn{5}{|l|}{ Pain intensity } \\
\hline Chinese participants & $8.78(1.12)$ & $0.96(1.21)$ & $8.58(1.64)$ & $1.13(1.12)$ \\
\hline Caucasian participants & $4.56(3.11)$ & $0.55(0.54)$ & $4.26(3.13)$ & $0.29(0.39)$ \\
\hline \multicolumn{5}{|l|}{ Self-unpleasantness } \\
\hline Chinese participants & $7.73(1.89)$ & $1.32(1.69)$ & 7.68 (1.82) & $1.25(1.40)$ \\
\hline Caucasian participants & $4.88(2.98)$ & $0.42(0.56)$ & $4.56(3.15)$ & $0.30(0.47)$ \\
\hline
\end{tabular}

related neural activity was modeled using a canonical hemodynamic response function.

Region-of-interest (ROI) analyses were conducted to test our hypothesis. The ROIs were defined based on an entirely independent data set that also compared needle penetration with Q-tip touch applied to neutral faces (Han et al., 2009). The ROI of the ACC was defined as a sphere with a radius of $10 \mathrm{~mm}$ centered at $x / y / z=4 / 40 / 38$ [MNI coordinates, Brodmann area (BA) 32/9]. ROIs of the left and right frontal cortices were defined as spheres with a radius of $10 \mathrm{~mm}$ centered at $-52 / 16 / 16$ (BA 44/45) and 52/22/20 (BA 45). The parameter estimates of signal intensity in association with the needle penetration and Q-tip touch applied to faces of the same or other races were calculated using Marsbar from both racial groups and subject to a repeated-measures ANOVA with Pain (painful vs nonpainful) and Group Membership (same vs other race) as within-subjects independent variables and Ethnicity (Caucasian vs Chinese participants) as a between-subjects variable.

Whole-brain statistical parametric mapping analyses were also performed to examine any other brain areas linked to the painful and nonpainful stimulation. Effects at each voxel were estimated and regionally specific effects were compared using linear contrasts in individual participants using a fixed effect analysis. One contrast (painful vs nonpainful stimulation) was calculated to define pain specific neural activations. Random effect analyses were then conducted across each participant group based on statistical parameter maps from each individual participant to allow population inference. Whole-brain statistical parametric mapping analyses were also calculated to confirm the interaction between Pain and Group Membership in each subject group by calculating the contrast $1-1-11$ (needle penetration applied to samerace faces, Q-tip applied to same-race faces, needle penetration applied to other-race faces, Q-tip applied to other-race faces). Given the previous hypothesis of brain activation related to empathy, significant activations were defined using a voxel threshold of $p<0.001$ and a spatial extent threshold of $k=50$.

\section{Results}

\section{Behavioral results}

Relative to Chinese participants, Caucasian participants scored higher on the Triandis individualism subscale $(4.86 \pm 0.48$ vs $\left.4.38 \pm 0.51, t_{(31)}=2.762, p<0.01\right)$ but lower on the collectivism subscale $\left(5.03 \pm 0.43\right.$ vs $\left.5.74 \pm 0.69, t_{(31)}=3.521, p<0.001\right)$. The mean ethnic identity scores were higher for Chinese than for Caucasians $\left(3.10 \pm 0.38\right.$ vs $\left.2.69 \pm 0.43, t_{(31)}=-2.874, p<0.01\right)$. Relative to Chinese participants, Caucasian participants showed higher rating scores of empathic concern $(25.6 \pm 3.26$ vs $22.6 \pm$ $\left.3.42, t_{(31)}=2.553, p=0.016\right)$, perspective-taking $(27.0 \pm 4.25 \mathrm{vs}$ $\left.20.2 \pm 3.43, t_{(31)}=5.083, p<0.001\right)$, and fantasy $(26.8 \pm 5.77 \mathrm{vs}$ $\left.19.6 \pm 3.44, t_{(31)}=4.359, p<0.001\right)$. No significant difference on the personal distress scale was observed between the two racial groups $\left(16.4 \pm 3.89\right.$ vs $\left.18.5 \pm 2.47, t_{(31)}=-1.852, p=0.074\right)$.

Response accuracy of the identification of painful and nonpainful stimuli during scanning was high and did not differ between the two racial groups (Caucasians: $94.0 \%$; Chinese: $93.2 \%$, $t=0.246 ; p>0.5)$. Rating scores of pain intensity and selfunpleasantness were higher for painful than non-painful stimulations $\left(F_{(1,31)}=156.82\right.$ and 107.544, both $p<0.001$ (see Table
1). Chinese scored higher in both pain intensity and selfunpleasantness than Caucasians $\left(F_{(1,31)}=35.645\right.$ and 20.187, $p<$ $0.001)$. Differential rating scores (painful vs non-painful stimuli) of pain intensity and self-unpleasantness were higher for Chinese than for Caucasians $\left(F_{(1,31)}=15.421\right.$ and 3.915, $p=0.001$ and 0.057 ) but did not differ between racial in-group and out-group members (both $p>0.1$ ).

\section{fMRI results}

The ROI analysis of signal intensity in the ACC confirmed increased activity to painful than non-painful stimulation $\left(F_{(1,31)}=\right.$ $7.876, p<0.01)$. Moreover, there was a significant interaction of Pain $\times$ Group Membership $\left(F_{(1,31)}=21.489, p<0.001\right)$, as ACC empathic responses were greater to racial in-group than outgroup members. However, the triple interaction of Pain $\times$ Group Membership $\times$ Ethnicity was not significant $\left(F_{(1,31)}=0.005, p=\right.$ $0.946)$, suggesting a similar patter of modulation of ACC empathic responses by racial group membership in Caucasian and Chinese participants. Post hoc analysis confirmed that, relative to the Q-tip touch, needle penetration increased the ACC activity when applied to racial in-group faces (Chinese: $t_{(16)}=2.73, p=$ 0.015 ; Caucasians: $\left.t_{(15)}=3.566, p=0.003\right)$ but not when applied to racial out-group faces (Chinese: $t_{(16)}=0.100, p=0.922$; Caucasians: $\left.t_{(15)}=0.030, p=0.977\right)$. Figure $1, c$ and $d$, shows contrast values to illustrate the effect of racial group membership on the ACC empathic responses. To assess whether one may predict ACC empathic responses to racial out-group members from ACC empathic responses to racial in-group members across individuals, we calculated correlation between ACC contrast values (painful vs non-painful) in association with racial in-group and out-group members and confirmed significant correlation between ACC empathic responses to racial in-group and out-group remembers $(r=0.469, p=0.006$, Fig. $1 e$ ).

ANOVAs of the signal intensity in the left frontal cortex showed a significant main effect of Pain $\left(F_{(1,31)}=4.847, p=\right.$ $0.035)$, suggesting enhanced left frontal activity to perceived painful than non-painful stimulation. Moreover, the empathic responses in the left frontal cortex was stronger in Caucasians than in Chinese, resulting in a reliable interaction of Pain $\times$ Ethnicity $\left(F_{(1,31)}=6.908, p=0.013\right)$. However, the left frontal empathic responses did not show reliable modulations by racial group membership $\left(F_{(1,31)}=2.353, p=0.135\right)$. Similar analyses of the right frontal activity failed to show any significant effect (all $p$ values $>0.1$ ).

Whole-brain statistical parametric mapping analyses further confirmed the results of ROI analyses. The contrast of painful versus non-painful stimulation applied to racial in-group faces indicated increased activations in the ACC/supplementary motor cortex (SMA) $(2 / 34 / 38, k=1205, Z=4.12)$, bilateral inferior frontal cortices $(-40 / 42 / 4, k=415, Z=3.72 ; 34 / 46 / 14, k=275$, $Z=3.91)$ and the left superior parietal cortex $(-48 /-50 / 52, k=$ $757, Z=5.25)$ in Caucasians and in the ACC/SMA $(-2 / 28 / 20$; 
$k=200, Z=3.65 ; 0 / 20 / 38, k=105, Z=2.94)$ and left inferior frontal/insula cortex $(-48 / 34 /-2, k=299, Z=3.48$, Fig. $1 f)$ in Chinese. The contrast of painful versus non-painful stimulation applied to racial out-group faces did not show any significant activation in both groups of subjects. The interaction analysis that compared the two contrasts (needle penetration vs Q-tip applied to racial in-group and out-group faces) revealed increased activation in the ACC (Caucasians: $4 / 28 / 42, k=243, Z=$ 3.37; Chinese: $-4 / 38 / 40, k=488, Z=3.27)$. The reverse comparison did not show any significant activation.

Finally, we calculated correlation between the magnitudes of empathic responses in the ACC/bilateral frontal cortex and subjective ratings of self-construals, empathy concern, and ethnic identity. However, we did not find any significant correlation results ( $p$ values $>0.05$ ).

\section{Discussion}

We showed that perception of painful stimulation applied to faces increased activity in parts (e.g., ACC and frontal/insula cortex) of the neural circuits underlying first-person pain experience, consistent with previous observations (Singer et al., 2004; Jackson et al., 2005; Gu and Han, 2007; Lamm et al., 2007; Saarela et al., 2007; Han et al., 2009). More interestingly, we found neuroimaging evidence for modulation of empathic neural responses by racial group membership, i.e., ACC empathic responses to perception of others in pain decreased remarkably when participants viewed faces of racial in-group members relative to racial out-group members. This effect was comparable in Caucasian and Chinese subjects and suggests that modulations of empathic neural responses by racial group membership are similar in different ethnic groups.

Our findings cannot be explained by different affective links between individuals since all models were strangers to our participants. Nor can the racial bias in empathic neural responses be accounted for by in-group advantage in emotion recognition (Elfenbein and Ambady, 2002) because empathic neural responses were defined by contrasting of painful and non-painful stimuli applied to faces with neutral expressions. As race helps defining in-group/out-group members (Cosmides et al., 2003), our fMRI results support the view that shared common membership enhances a perceiver's empathic concerns for others (Hornstein, 1978). Empathy consists of both affective components (e.g., emotional sharing) and cognitive components (e.g., perspective taking) (Decety and Jackson, 2006; Fan and Han, 2008) and the ACC mainly contributes to the affective component of empathy (Singer et al., 2004). Thus the own-race bias in ACC activity linked to empathy for pain may mediate enhanced sharing of feelings and emotions of ethnic in-group members. However, such neural empathic bias toward racial in-group members did not necessarily result in different conscious subjective ratings of others' pain intensity and induced self-unpleasantness related to racial in-group and out-group members, as indicated by measures of subjective ratings in the current work. Thus it is likely that the own-race bias in empathy-related ACC activity observed here reflected unconscious affective response to racial in-group members. In contrast, as the empathy-related lateral frontal activity covaried with task demands [i.e., being present in a pain judgment task but absent in a counting task related to painful stimuli (Gu and Han, 2007)] and co-occurred with subjective reports in the current work, it may be speculated that the lateral frontal cortex is involved in conscious cognitive evaluations of others' pain.

Although our fMRI results suggest own-race bias in empathy- related ACC activity, the results of correlation analysis indicate that empathic neural responses to in-group and out-group members were not independent. Participants who showed greater empathic neural responses to in-group members also showed stronger empathic neural responses to out-group members, reflecting individual differences in general ability of empathy.

It should be noted that not any in-group membership results in enhancement of empathy. For example, university group status (shared vs unshared) does not have an impact on empathetic induction (Batson et al., 1997b). Racial group membership defines coalitions and alliances during evolution (Cosmides et al., 2003) and thus results in strong modulation of the neural substrates of emotional components of empathy. Our questionnaire measurements suggested difference in cultural values, ethnic identity, and empathy ability between the two participant groups. However, they showed similar neural substrates of empathy modulation by racial group membership. It appears that, relative to cultural influence on empathy, if any, the modulation of empathy by racial group membership is more fundamental and plays a more pivotal role in shaping social behaviors.

Our results complement previous observations that empathic neural responses are modulated by affective link between individuals (Singer et all, 2006), personal experience (Cheng et al., 2007), and task demand and stimulus reality (Fan and Han, 2008; $\mathrm{Gu}$ and Han, 2007) by showing modulation of empathic neural responses by social relationship. Our findings have significant implications for understanding real-life social behaviors and provide a neurocognitive mechanism for stronger intentions to help racial in-group than out-group members (Gaertner and Dovidio, 1977).

\section{References}

Batson CD (1991) The altruism question: toward a social-psychological answer. Hillsdale, NJ: Lawrence Erlbaum.

Batson CD, Polycarpou MP, Harmon-Jones E, Imhoff HJ, Mitchener EC, Bednar LL, Klein TR, Highberger L (1997a) Empathy and attitudes: can feeling for a member of a stigmatized group improve feelings toward the group? J Pers Soc Psychol 72:105-118.

Batson CD, Sager K, Garst E, Kang M, Rubchinsky K, Dawson K (1997b) Is empathy-induced helping due to self-other merging? J Pers Soc Psychol 73:495-509.

Botvinick M, Jha AP, Bylsma LM, Fabian SA, Solomon PE, Prkachin KM (2005) Viewing facial expressions of pain engages cortical areas involved in the direct experience of pain. Neuroimage 25:312-319.

Cheng Y, Lin CP, Liu HL, Hsu YY, Lim KE, Hung D, Decety J (2007) Expertise modulates the perception of pain in others. Curr Biol 17:1708-1713.

Cosmides L, Tooby J, Kurzban R (2003) Perceptions of race. Trends Cogn Sci 7:173-179.

Davis MH (1996) Empathy: a social psychological approach. Boulder: Westview.

Decety J, Jackson PL (2006) A social-neuroscience perspective on empathy. Curr Dir Psychol Sci 15:54-58.

de Waal FBM (2008) Putting the altruism back into altruism: the evolution of empathy. Annu Rev Psychol 59:279-300.

Elfenbein HA, Ambady N (2002) On the universality and cultural specificity of emotion recognition: A meta-analysis. Psychol Bull 128:203-235.

Fan Y, Han S (2008) Temporal dynamic of neural mechanisms involved in empathy for pain: an event-related brain potential study. Neuropsychologia 46:160-173.

Gaertner SL, Dovidio JF (1977) The subtlety of white racism, arousal, and helping behavior. J Pers Soc Psychol 35:691-707.

Gu X, Han S (2007) Attention and reality constraints on the neutral processes of empathy for pain. Neuroimage 36:256-267.

Han S, Fan Y, Xu X, Qin J, Wu B, Wang X, Aglioti SM, Mao L (2009) Empathic neural responses to others' pain are modulated by emotional contexts. Hum Brain Mapp, in press.

Hornstein HA (1978) Promotive tension and prosocal behavior: a Lewinian 
analysis. In Altruism, sympathy, and helping: psychological and sociological principles (Wispe L, ed), pp 177-207. New York: Academic.

Jackson PL, Meltzoff AN, Decety J (2005) How do we perceive the pain of others? A window into the neural processes involved in empathy. Neuroimage 24:771-779.

Johnson JD, Simmons CH, Jordan A, MacLean L, Taddei J, Thomas D (2002) Rodney King and O. J. revisited: The impact of race and defendant empathy induction on judicial decisions. J Appl Soc Psychol 32:1208-1223.

Lamm C, Batson CD, Decety J (2007) The neural substrate of human empathy: effects of perspective-taking and cognitive appraisal. J Cogn Neurosci 19:42-58.

Phinney JS (1992) The Multigroup Ethnic Identity Measure: a new scale for use with diverse groups. J Adoles Res 7:156-176.
Preston SD, de Waal FB (2002) Empathy: its ultimate and proximate bases. Behav Brain Sci 25:1-20.

Saarela MV, Hlushchuk Y, Williams AC, Schürmann M, Kalso E, Hari R (2007) The compassionate brain: humans detect intensity of pain from another's face. Cereb Cortex 17:230-237.

Singer T, Seymour B, O’Doherty J, Kaube H, Dolan RJ, Frith CD (2004) Empathy for pain involves the affective but not sensory components of pain. Science 303:1157-1162.

Singer T, Seymour B, O’Doherty JP, Stephan KE, Dolan RJ, Frith CD (2006) Empathic neural responses are modulated by the perceived fairness of others. Nature 439:466-469.

Triandis HC, Gelfand MJ (1998) Converging measurement of horizontal and vertical individualism and collectivism. J Pers Soc Psychol 74:118-128. 